\title{
AN UPPER BOUND FOR THE LEAST DILATATION
}

\author{
MAX BAUER
}

\begin{abstract}
We given an upper bound for the least dilatation arising from a pseudo-Anosov map of a closed surface of genus greater or equal to three.
\end{abstract}

\section{INTRODUCTION AND BACKGROUND}

Introduction. Throughout the paper, $F=F_{g}$ will be a closed surface of genus $g$ with negative Euler characteristic. Suppose that $(\mathscr{F}, \nu)$ is a measured foliation (see [FLP]) and $\phi$ is an orientation preserving homeomorphism of $F$, then we define $\phi(\mathscr{F})$ to be the foliation whose leaves are the images of the leaves of $\mathscr{F}$. Furthermore, $\phi_{*}(\nu)$ is a measure on $\phi(\mathscr{F})$ that is defined as the push forward of the measure $\nu$ under $\phi$. To be more explicit, if $\alpha$ is an arc transverse to the foliation $\phi(\mathscr{F})$, then $\phi_{*}(\nu)(\alpha)=\nu\left(\phi^{-1}(\alpha)\right)$. We define $\bar{\phi}(\mathscr{F}, \nu)=$ $\left(\phi(\mathscr{F}), \phi_{*}(\nu)\right)$.

An orientation preserving homeomorphism $\phi$ of $F$ is pseudo-Anosov (or p.A.) if there is a pair of transverse arational (i.e. no closed leaves) measured foliations $(\mathscr{F}, \nu)$ and $\left(\mathscr{F} \perp, \nu^{\perp}\right)$ in $F$, such that $\bar{\phi}(\mathscr{F}, \nu)=(\mathscr{F}, \lambda \nu)$ and $\bar{\phi}\left(\mathscr{F} \perp, \nu^{\perp}\right)=\left(\mathscr{F} \perp,(1 / \lambda) \nu^{\perp}\right)$, for some $\lambda>1$. $\lambda$ is called the dilatation of $\phi$, and we define the 'spectrum' of $F$ as

$$
\operatorname{Spec}(F)=\{\log \lambda: \lambda \text { is the dilatation of a p.A. self-map of } F\} \subset \mathbb{R} .
$$

$\operatorname{Spec}(F)$ has a geometric interpretation as the collection of Teichmüller distances between Riemann surfaces of the same topological type as $F$ (see [Ab]). Furthermore, a pseudo-Anosov map $\phi$ realizes the smallest topological entropy in its homotopy class and the topological entropy of $\phi$ is given by the logarithm of its dilatation (see [FLP]). It is known that $\operatorname{Spec}(F)$ is discrete (see [AY]).

The main goal of this note is to show that the smallest element $\delta_{g}$ of $\operatorname{Spec}\left(F_{g}\right)$ allows the upper bound

$$
\delta_{g} \leq \frac{\log 6}{g}, \quad \text { for all } g \geq 3 .
$$

This is an improvement of the upper bound given in [P2].

Background. We begin by establishing some notation. The group of $l \times l$ matrices over $\mathbb{Z}$ is denoted by $\operatorname{Mat}_{l}(\mathbb{Z})$, and the spectrum of $A \in \operatorname{Mat}_{l}(\mathbb{Z})$ is the set of eigenvalues of $A$ listed with multiplicity. We say that an eigenvalue $\lambda$ in

Received by the editors January 12, 1990.

1980 Mathematics Subject Classification (1985 Revision). Primary 57N05.

Partially supported by the N.S.F. 
the spectrum of $A$ has maximum modulus if the modulus of $\lambda$ strictly exceeds the modulus of any other element of the spectrum of $A$. Suppose next that $A$ is an $n \times m$ matrix with $n, m \geq 1$, then by an expression of the form $A>0$, $A \geq 0$, etc. we mean that the relevant relation holds for each component of $A$. A matrix $A \in \operatorname{Mat}_{l}(\mathbb{Z})$ is said to be Perron-Frobenius or P.F. if $A \geq 0$ and for some $n \geq 1$ we have $A^{n}>0$.

Perron-Frobenius matrices have the following well known spectral properties (see $[\mathrm{Ga}])$.

Theorem (Perron-Frobenius). The spectrum of a P.F. matrix $A$ contains an element $\lambda$ of maximum modulus that is positive real with corresponding eigenvector $x^{*}$ strictly positive. $x^{*}$ is the unique positive eigenvector and $\lambda$ is a simple root of the characteristic polynomial of $A$.

The main tool in the present investigation is the theory of train tracks as introduced by Thurston [Th]. The material that is needed for the present investigation is collected in the section on train tracks in [Ba]. We refer the reader who wishes more information on surface homeomorphisms and train tracks to [CB, FLP, Th, Pa, PP] and the monograph on train tracks [HP]. We remark in particular that if $\tau$ is a train track embedded in the surface $F$, and if $V(\tau)$ denotes the set of measures on $\tau$, then there is an embedding $\mathscr{I}$ of $V(\tau)$ into the space of (equivalence classes of) measured foliations $\mathscr{M} \mathscr{F}(F)$. Moreover, if $\tau$ is invariant under a homeomorphism $\phi$ of $F$, then there are induced maps $\hat{\phi}: V(\tau) \rightarrow V(\tau)$ and $\bar{\phi}: \mathscr{M F}(F) \rightarrow \mathscr{M} \mathscr{F}(F)$ such that $\mathscr{I} \circ \hat{\phi}=\bar{\phi} \circ \mathscr{I}$.

\section{AN UPPER BOUND BY EXAMPLE}

A class of pseudo-Anosov maps. We describe in this section the map that yields the upper bound mentioned in the introduction.

If $k$ is a simple closed curve embedded in $F$, then we denote the (right handed) Dehn twist along $k$ by $\tau_{k}$. (For a definition of Dehn twist see for example [Ba].) For $g \geq 3$, we take the surface $F_{g}$ as a sphere with $g$ handles attached so that $F_{g}$ is invariant under the rotation $\rho=\rho_{g}$ by $2 \pi / g$ about the axis $L$ through the north and south pole of the sphere. Figure 1 shows the case $g=3$. We then define

$$
\psi=\psi_{g}=\rho \circ \tau_{c}^{-1} \circ \tau_{b} \circ \tau_{a} .
$$

Whenever the dependency of an object on the genus $g$ is clear we will simplify notation by suppressing this dependency. For example the maps $\rho, \tau_{c}^{-1}, \tau_{b}$ and $\tau_{a}$ depend on $g$.

Our first goal is to show that $\psi_{g}$ is (isotopic to) a pseudo-Anosov homeomorphism using the following pseudo-Anosov recognition theorem (see [CB]).

Theorem (Casson). A homeomorphism $\phi$ of $F$ is isotopic to a p.A. map if there is a $\phi$ invariant train track $\tau$ that fills $F$, such that no proper subtrack $\tau^{\prime}$ of $\tau$ is invariant under $\phi$, and such that if $\tau$ itself is a subtrack of a $\phi$ invariant train track $\tau^{\prime \prime}$ (not necessarily proper) then the induced map $\phi^{\prime \prime}: V\left(\tau^{\prime \prime}\right) \rightarrow V\left(\tau^{\prime \prime}\right)$ has no nonzero fixed point.

The train track that will allow us to apply the previous theorem is $\tau(g)$ as shown in Figure 2 in case $g=3$. It is clear how $\tau(g)$ is defined (up to isotopy) for $g \geq 3$. We isotope $\tau(g)$ so that $\tau(g)$ is invariant under $\rho$. Note that 


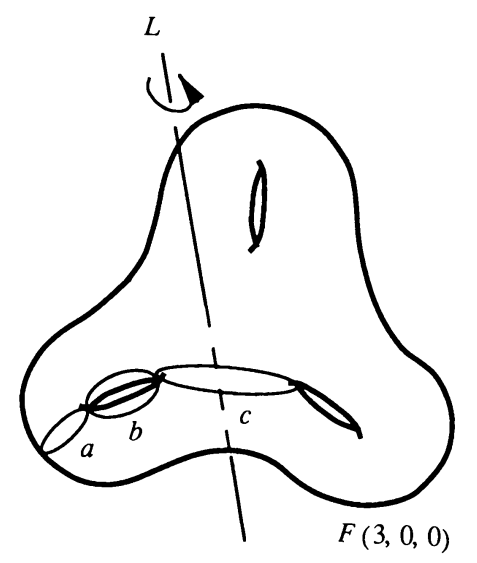

FiguRe 1. The surface $F_{g}$, in case $g=3$

$\tau(g)$ has as complementary regions $2 g$ tri-gons and $2 g$-gons, hence $\tau(g)$ fills the surface. The number of branches of $\tau(g)$ is $7 g$.

We need to be more precise as to how $\psi_{g}$ acts on $F_{g}$ and to that end we use the notation for branches of $\tau(g)$ as illustrated in Figure 2 in case $g=3$. Clearly we can isotope $\tau_{b} \circ \tau_{a}$ so that it fixes the switches of $\tau(g)$ and all of the branches except for $b_{1}, b_{2}$, and $b_{3}$. We also isotope $\tau_{c}^{-1}$ such that it fixes the switches of $\tau(g)$ and only moves the branches $b_{4}$ and $b_{5}$.

By choosing the central ties of a standard tie neighborhood of $\tau(g)$ carefully, we may assume that $\tau_{b} \circ \tau_{a}\left(b_{1}\right)$ intersects the central ties corresponding to the branches of $\left\{\rho^{g-1}\left(b_{5}\right), \rho^{g-1}\left(a_{2}\right), b_{3}, a_{1}, b_{4}\right\}$; also $\tau_{b} \circ \tau_{a}\left(b_{2}\right)$ intersects the central tie corresponding to the branch $b_{1}$, and $\tau_{b} \circ \tau_{a}\left(b_{3}\right)$ intersects the central ties corresponding to the branches of $\left\{\rho^{g-1}\left(b_{4}\right), b_{2}, b_{5}\right\}$. Similarly, we may assume that $\tau_{c}^{-1}\left(b_{4}\right)$ intersects the central ties corresponding to the branches of $\left\{\rho\left(b_{3}\right), a_{2}, b_{3}, a_{1}, b_{4}\right\}$, and $\tau_{c}^{-1}\left(b_{5}\right)$ intersects the ones corresponding to $\left\{b_{3}, a_{1}, \rho\left(b_{3}\right), a_{2}, b_{5}\right\}$.

For future reference, we summarize

Lemma 1. For $g \geq 3, \tau(g)$ is a $\psi_{g}$ invariant train track that fills $F_{g}$.

Note that if we define $\bar{B}=\bigcup_{i=0}^{g-1} B_{i}$, where $B_{i}=\left\{\rho^{i}\left(b_{1}\right), \ldots, \rho^{i}\left(b_{5}\right)\right\}$, for $0 \leq i \leq g-1$, then $\tau_{c}^{-1} \circ \tau_{b} \circ \tau_{a}$ only affects the branches $B_{0}$. Whenever we need to assume that the branches of $\tau(g)$ are ordered, we choose the following ordering

$$
\begin{array}{r}
\left(\rho^{0}\left(b_{1}\right), \ldots, \rho^{0}\left(b_{5}\right), \ldots, \rho^{g-1}\left(b_{1}\right), \ldots, \rho^{g-1}\left(b_{5}\right),\right. \\
\left.\rho^{0}\left(a_{1}\right), \rho^{0}\left(a_{2}\right), \ldots, \rho^{g-1}\left(a_{1}\right), \rho^{g-1}\left(a_{2}\right)\right) .
\end{array}
$$

Note that this defines an induced ordering of the branches $\bar{B}$.

If $\phi$ is a composition of $\rho, \tau_{c}^{-1}$, and $\tau_{b} \circ \tau_{a}$, then we define $\operatorname{Inc}(\phi)$ to be the incidence matrix of $\phi$ with respect to the ordering of the branches of $\tau$ specified above. We remark that $\operatorname{Inc}(\phi)$ is well defined by our convention of how $\rho, \tau_{c}^{-1}$, and $\tau_{b} \circ \tau_{a}$ act on $\tau(g)$. Note that the distributive property of the 'hat' operator (i.e. $\left.\widehat{\phi}_{1} \phi_{2}=\hat{\phi}_{1} \hat{\phi}_{2}\right)$ implies that for example $\operatorname{Inc}\left(\tau_{c}^{-1} \circ \tau_{b} \circ \tau_{a}\right)=$ $\operatorname{Inc}\left(\tau_{c}^{-1}\right) \operatorname{Inc}\left(\tau_{b} \circ \tau_{a}\right)$.

The following lemma is crucial in showing that $\psi_{g}$ is p.A. 


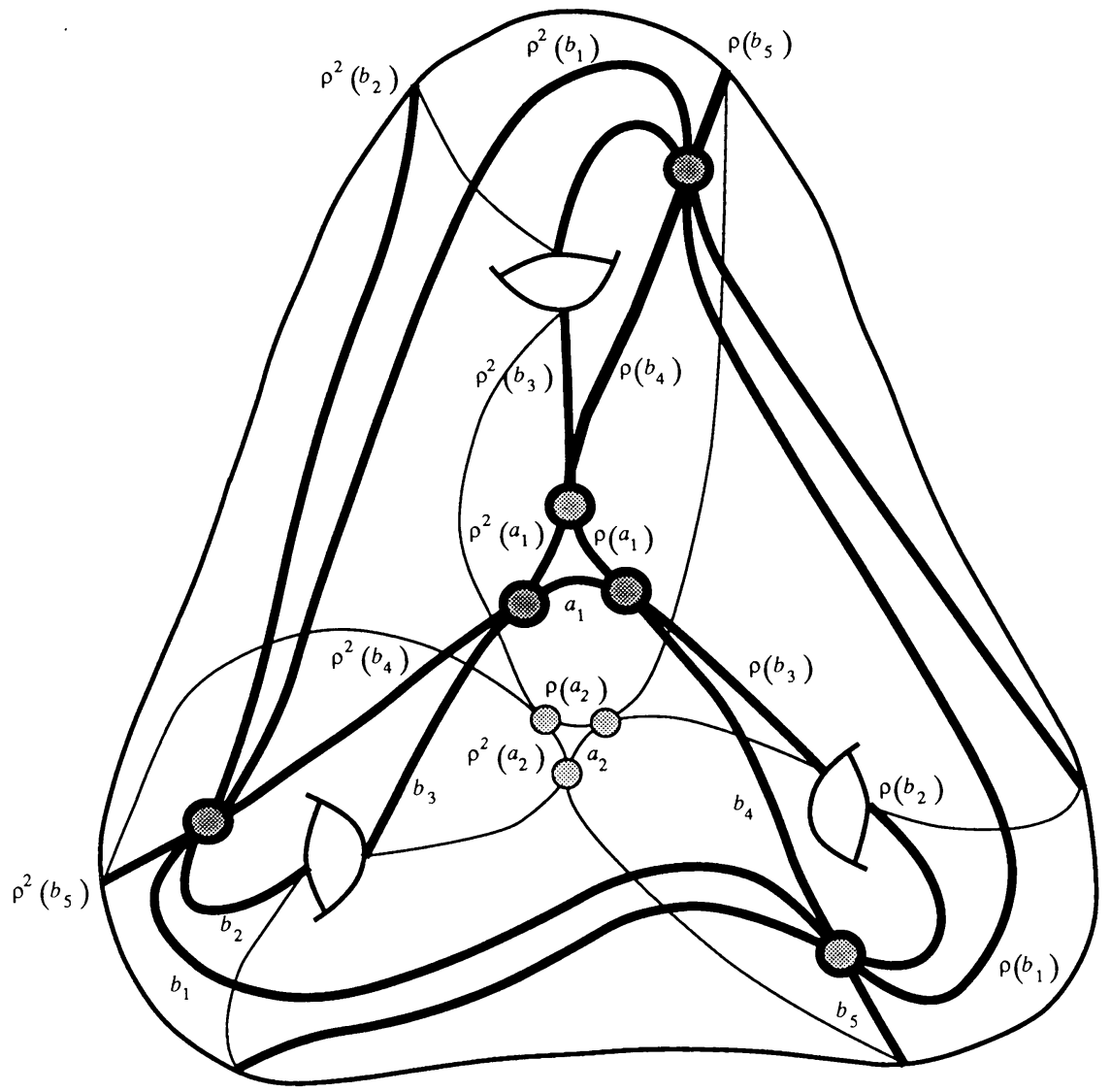

FIGURE 2. Invariant train track for $\psi_{g}$, in case $g=3$

Lemma 2. If $w^{(0)} \in \mathbb{R}^{7 g}$ represents a nonzero weight on $\tau(g)$ (we do not require that the switch conditions hold), such that $w^{(0)}$ is positive on at least one branch of $\bar{B}$, then there is an $n_{0}$ such that for $n>n_{0}, \operatorname{Inc}\left(\psi^{n}\right) w^{(0)}>0$.

Proof. Recall that $\tau_{c}^{-1} \circ \tau_{b} \circ \tau_{a}$ only moves the branches $B_{0}$. This will be most important and used repeatedly without being mentioned.

Claim 1. Suppose that $T \in \operatorname{Mat}_{5}(\mathbb{Z})$ consists of those rows and columns of the incidence matrix $\operatorname{Inc}\left(\tau_{c}^{-1} \circ \tau_{b} \circ \tau_{a}\right)$ that correspond to the branches $B_{0}$, then $T^{4}>0$.

We readily see that

and compute.

$$
T=\left(\begin{array}{lllll}
0 & 1 & 0 & 0 & 0 \\
0 & 0 & 1 & 0 & 0 \\
2 & 0 & 1 & 1 & 1 \\
1 & 0 & 0 & 1 & 0 \\
0 & 0 & 1 & 0 & 1
\end{array}\right)
$$

Claim 2. If $w \in \mathbb{R}^{7 g}$ represents a weight on $\tau(g)$ that is positive on a set $B_{0}^{\prime} \subset B_{0}$, then the weight $\operatorname{Inc}\left(\psi^{g-1} \circ \rho\right) w$ is also positive on $B_{0}^{\prime}$.

This is immediate. 
Claim 3. If for some $0 \leq t \leq g-1, w \in \mathbb{R}^{7 g}$ is a weight that is positive on all branches of $B_{t}$, then $\operatorname{Inc}\left(\psi^{g}\right) w$ is also positive on the branches $B_{t}$.

Indeed, $w^{\prime}=\operatorname{Inc}\left(\psi^{g-t}\right) w$ is positive on the branches $B_{0}$, and as each row of $T$ contains at least one positive entry, $w^{\prime \prime}=\operatorname{Inc}\left(\tau_{c}^{-1} \circ \tau_{b} \circ \tau_{a}\right) w^{\prime}$ is still positive on the branches $B_{0}$. But then $\operatorname{Inc}\left(\psi^{t-1} \rho\right) w^{\prime \prime}$ is positive on $B_{t}$ as desired.

Claim 4. If $w$ is a weight that is positive on the branches of $B_{0}$, then $w^{*}=$ $M\left(\tau_{c}^{-1} \circ \tau_{b} \circ \tau_{a}\right) w$ is positive on $B_{0} \cup\left\{a_{1}, a_{2}\right\}$.

Indeed, we already remarked in the proof of Claim 3 that $w^{*}$ is positive on branches of $B_{0}$, and the fact that $w^{*}$ is positive on the branches $a_{1}$ and $a_{2}$ can be seen directly from our convention of how $\tau_{c}^{-1}$ and $\tau_{b} \circ \tau_{a}$ acts on $\tau(g)$.

We now take $w^{(0)}$ as in the statement of the lemma, hence there exists $t \in\{0, \ldots, g-1\}$, such that $w^{(0)}$ is positive on a branch of $B_{t}$.

We first prove that $\operatorname{Inc}\left(\psi^{n}\right) w^{(0)}$, for $n$ large enough, is positive on the branches of $\bar{B}$. The proof is by induction on the number of $i$ such that $\operatorname{Inc}\left(\psi^{n}\right) w^{(0)}$, for $n$ large enough, is positive on the branches of $B_{i}$. We take the subscripts of $B_{i}$ as elements of the cyclic group on $g$ elements.

For the basis step, we remark that $w^{(1)}=\operatorname{Inc}\left(\psi^{g-t}\right) w^{(0)}$ is positive on a branch of $B_{0}$. It follows from Claim 1 and Claim 2 that $w^{(2)}=\operatorname{Inc}\left(\psi^{4 g}\right) w^{(1)}$ is positive on all branches of $B_{0}$.

For the induction step we assume that for some $n, w^{(3)}=\operatorname{Inc}\left(\psi^{n}\right) w^{(0)}$ is positive on the branches of $B_{0}, \ldots, B_{j}$, for some $j \in\{0, \ldots, g-1\}$. We first note that $w^{(4)}=\operatorname{Inc}\left(\psi^{g-j}\right) w^{(3)}$ is positive on the branches of $B_{0}$. But then $w^{(5)}=\operatorname{Inc}\left(\tau_{c}^{-1} \circ \tau_{b} \circ \tau_{a}\right) w^{(4)}$ is positive on the branches $B_{0} \cup\left\{\rho\left(b_{3}\right)\right\}$ (as follows from our convention of how $\psi_{g}$ acts on $\left.\tau(g)\right)$, hence $w^{(6)}=\operatorname{Inc}\left(\psi^{g-2} \rho\right) w^{(5)}$ is positive on the branches $B_{g-1} \cup\left\{b_{3}\right\}$. Using the fact that $w^{(6)}$ is positive on a branch of $B_{0}$, we see as above, using Claim 1 and Claim 2 , that $w^{(7)}=$ $\operatorname{Inc}\left(\psi^{4 g}\right) w^{(6)}$ is positive on all branches of $B_{0}$. We conclude that $w^{(8)}=$ $\operatorname{Inc}\left(\psi^{j+1}\right) w^{(7)}$ is positive on the branches of $B_{j+1}$. To complete the induction step, note first that $w^{(8)}=\operatorname{Inc}\left(\psi^{6 g}\right) w^{(3)}$. As $w^{(3)}$ is positive on the branches of $B_{0}, \ldots, B_{j}$, so is $w^{(8)}$ as follows from Claim 3 .

We showed that for some $m \geq 1$ we have that $\operatorname{Inc}\left(\psi^{m}\right) w^{(0)}$ is positive on the branches of $\bar{B}$. To finish the proof of the lemma, we use Claim 4 to see that $\operatorname{Inc}\left(\psi^{m+g}\right) w^{(0)}$ is positive on all branches of $\tau$ and the claim follows by taking $n_{0}=m+g$. Q.E.D.

In order to show that $\psi_{g}$ is pseudo-Anosov, we need to analyze the incidence matrix $\operatorname{Inc}\left(\psi_{g}\right)$. We claim

Lemma 3. (a)

$$
\operatorname{Inc}\left(\psi_{g}\right)=\left(\begin{array}{cc}
S_{g} & \overline{0} \\
C & P
\end{array}\right) \in \operatorname{Mat}_{7 g}(\mathbb{Z}),
$$

where $\overline{0}$ is the $5 g \times 2 g$ zero matrix and $P \in \operatorname{Mat}_{2 g}(\mathbb{Z})$ is a permutation matrix. (Of course $C$ is a $2 g \times 5 g$ matrix.) Moreover,

(b) the 'small' incidence matrix $S=S_{g} \in \operatorname{Mat}_{5 g}(\mathbb{Z})$ of $\psi$ with respect to the branches $\bar{B}$ of $\tau(g)$ is P.F.

Proof. For the proof of part (a) we remark that $\rho$ and hence $\psi$ induces a permutation of the branches $A=\left\{\rho^{i}\left(a_{j}\right): 0 \leq i \leq g-1\right.$ and $\left.j=1,2\right\}$. To be more specific, if $w$ is a weight on $\tau(g)$ that is positive on a single branch 
$a$ of $A$, then $\operatorname{Inc}(\psi) w$ is positive only on $\rho(a)$. This explains the subblocks $\overline{0}$ and $P$ of $\operatorname{Inc}(\psi)$.

For the proof of part (b) we define $e_{i} \in \mathbb{R}^{5 g}$ to be the $i$ th unit vector in $\mathbb{R}^{5 g}$. To show that $S$ is P.F. it is of course enough to show that there exists an $n \geq 1$ such that for $1 \leq i \leq 5 g$ we have $S^{n} e_{i}>0$. If for $1 \leq i \leq 5 g$, we define $\bar{e}_{i} \in \mathbb{R}^{7 g}$ to be the $i$ th unit vector, then, using part (a), we readily see that it is enough to show that there exists an $n$ such that for $1 \leq i \leq 5 g$ we have that $\operatorname{Inc}\left(\psi^{n}\right) \bar{e}_{i}$ is positive on branches of $\bar{B}$. This, however, follows from Lemma 2. Q.E.D.

We can now prove

Proposition 4. For $g \geq 3, \psi_{g}$ is pseudo-Anosov.

Proof. We need to check the conditions of Casson's theorem.

The first two conditions, namely that $\tau(g)$ fills $F_{g}$ and is invariant under $\psi_{g}$ follow from Lemma 1 .

To show the third condition, suppose that $\tau^{\prime}=\tau^{\prime}(g)$ is a subtrack of $\tau=\tau(g)$ that is invariant under $\psi_{g}$. We will show that $\tau^{\prime}$ cannot be a proper subtrack of $\tau(g)$.

We choose a nonzero measure $\mu^{\prime}$ on $\tau^{\prime}$ and extend $\mu^{\prime}$ to a nonzero measure $\mu \in V(\tau)$ by defining $\mu(b)=\mu^{\prime}(b)$, if $b$ is a branch of $\tau^{\prime}$ and $\mu(b)=0$, otherwise. It is easy to see that any nonzero measure on $\tau$ is positive on some branch of $\bar{B}$, hence by Lemma 2 , we can find $n>0$ such that $\hat{\psi}^{n}(\mu)$ is positive on all branches of $\tau$. ( $\hat{\psi}$ being the self-map of $V(\tau)$ that is induced by $\psi$. .) As we assumed that $\tau^{\prime}$ is invariant under $\psi$, and as $\mu$ is zero on branches of $\tau \backslash \tau^{\prime}$, the measure $\hat{\psi}^{n}(\mu)$ must also be zero on these branches. It follows that $\tau^{\prime}=\tau$ as desired.

We are left to check the last condition in Casson's theorem, namely if $\tau$ is a subtrack of a $\psi$ invariant train track $\tau^{\prime \prime}=\tau^{\prime \prime}(g)$, then the induced map $\hat{\phi}^{\prime \prime}: V\left(\tau^{\prime \prime}\right) \rightarrow V\left(\tau^{\prime \prime}\right)$ has no nonzero fixed point. An Euler characteristic argument shows that for $\tau^{\prime \prime}$ to satisfy the fourth condition in the definition of train track, the branches of $\tau^{\prime \prime} \backslash \tau$ must be contained in the closure of the two complementary $g$-gons of $\tau$. But as $\psi$ induces a rotation of these complementary $g$-gons, we readily see that $\tau^{\prime \prime}$ can only be $\psi$ invariant and satisfy the fourth condition in the definition of train track if $\tau^{\prime \prime}=\tau$.

Assume now to derive a contradiction that $\mu \in V(\tau)$ is a nonzero fixed point of $\hat{\psi}: V(\tau) \rightarrow V(\tau)$, hence we have a nonzero fixed point $x \in \mathbb{R}^{7 g}$ of $\operatorname{Inc}(\psi)$. As remarked above, any nonzero measure on $\tau$ is positive on some branch of $\bar{B}$, hence Lemma 2 applies to show that $x>0$. It follows from Lemma 3 that $x$ restricts to a nonzero fixed point $\bar{x}$ of $S$. But as $S \in \operatorname{Mat}_{5 g}(\mathbb{Z})$ is P.F. and integral, $S^{n} \geq 1$, for $n$ large enough, and as $5 g>1$, we see that $S$ cannot have a nonzero fixed point. This contradiction shows that our assumption of $\hat{\psi}$ having a nonzero fixed point was absurd.

We showed that all the conditions in Casson's theorem are satisfied and conclude that $\psi_{g}$ is indeed p.A. Q.E.D.

The upper bound. We will show

Proposition 5. For $g \geq 3$, the dilatation $\lambda_{g}$ of $\psi_{g}$ satisfies

$$
\log \lambda_{g} \leq \frac{\log 6}{g}
$$


As a corollary we get our main result.

Theorem 6. The smallest element $\delta_{g}$ in $\operatorname{Spec}\left(F_{g}\right)$ satisfies

$$
\delta_{g} \leq \frac{\log 6}{g}, \quad \text { for } g \geq 3 .
$$

Before we prove the proposition some remarks are in order.

Remark. (a) One can define $F_{g}$ and $\phi_{g}$, for $g=2$, but a computation shows that the dilatation $\lambda_{2}$ of $\psi_{2}$ satisfies $\lambda_{2}^{2} \approx 6.018>6$.

(b) [P2] shows, using [P1], that $\rho \circ \tau_{c} \circ \tau_{b}^{-1} \circ \tau_{a}$ is p.A. with dilatation $l_{g}$ that satisfies $l_{g} \leq(\log 11) / g$. The fact that $\psi_{g}$ is p.A. does not follow from [P1] as we perform right-handed Dehn twists along two curves that intersect. The method in this note can be extended to produce a large class of p.A. maps. (See [Ba].)

\section{Proof of Proposition 5.}

Step 1. We choose $g \geq 3$ and show that we can find the dilatation of $\psi=\psi_{g}$ by spectrally analyzing the incidence matrix $\operatorname{Inc}(\psi)$. This is a standard technique. We then proceed to demonstrate that the dilatation of $\psi$ is in fact given by the spectral radius of the smaller P.F. matrix $S=S_{g}$ (as in Lemma 3).

If we identify $V(\tau)$ with a closed cone in $\mathbb{R}^{7 g}$, then $(V(\tau) \backslash\{0\}) / \mathbb{R}_{+}$, where $\mathbb{R}_{+}$denotes the positive real numbers, can be represented by a closed cell. $\operatorname{Inc}(\psi)$ induces a continuous self-map of this cell and we conclude from the Brouwer fixed point theorem that there exist $\sigma=\sigma_{g}>0$ and $x \in \mathbb{R}^{7 g}$ such that $\operatorname{Inc}(\psi) x=\sigma x$. Moreover, $x$ corresponds to a nonzero measure on $\tau$.

We show next that $\sigma>1$ and that $\sigma$ is the spectral radius of $S$. Indeed, we conclude from Lemma 3 that if we define $\bar{x} \in \mathbb{R}^{5 g}$ to be the restriction of $x$ onto its first $5 g$ coordinates, then $S \bar{x}=\sigma \bar{x}$. Moreover, $x>0$, as follows from Lemma 2, and hence $\bar{x}>0$. The uniqueness statement of the PerronFrobenius theorem shows that $\sigma$ is the spectral radius of $S$. As $S$ is P.F. and integral, we conclude that $\sigma>1$.

We claim that $\sigma$ equals the dilatation $\lambda=\lambda_{g}$ of $\psi_{g}$, and to that end we define $\mu \in V(\tau)$ to be the measure that corresponds to $x \in \mathbb{R}^{7 g}$. We further define $(\mathscr{F}, \nu)=\mathscr{I}(\mu)$. We showed above that $\operatorname{Inc}(\psi) x=\sigma x$, hence $\hat{\psi} \mu=\sigma \mu$. As $\mathscr{J} \circ \hat{\psi}=\bar{\psi} \circ \mathscr{I}$, we conclude that $\bar{\psi}(\mathscr{F}, \nu)=(\mathscr{F}, \sigma \nu)$. But there is only one such foliation (class) for a p.A. map with $\sigma>1$, hence $\sigma=\lambda$ as desired. The claim follows.

Step 2. We need to bound above the spectral radius $\lambda$ of $S$. To that end we think of the matrix $S$ and its powers as having $g$ columns, the $g$ elements of each column being $5 \times 5$ matrices. We find

$$
S=\left(\begin{array}{cccccccc}
A_{1} & \overline{0} & \overline{0} & \overline{0} & \cdots & \overline{0} & \overline{0} & I \\
A_{2} & \overline{0} & \overline{0} & \overline{0} & \cdots & \overline{0} & \overline{0} & \overline{0} \\
A_{3} & I & \overline{0} & \overline{0} & \cdots & \overline{0} & \overline{0} & \overline{0} \\
\overline{0} & \overline{0} & I & \overline{0} & \cdots & \overline{0} & \overline{0} & \overline{0} \\
& & & & \vdots & & & \\
\overline{0} & \overline{0} & \overline{0} & \overline{0} & \cdots & \overline{0} & I & \overline{0}
\end{array}\right) \in \operatorname{Mat}_{5 g}(\mathbb{Z})
$$


where

$$
\begin{gathered}
A_{1}=\left(\begin{array}{lllll}
0 & 0 & 0 & 0 & 0 \\
0 & 0 & 0 & 0 & 0 \\
0 & 0 & 0 & 0 & 0 \\
0 & 0 & 1 & 0 & 0 \\
1 & 0 & 0 & 0 & 0
\end{array}\right), \quad A_{2}=\left(\begin{array}{llllll}
0 & 1 & 0 & 0 & 0 \\
0 & 0 & 1 & 0 & 0 \\
2 & 0 & 1 & 1 & 1 \\
1 & 0 & 0 & 1 & 0 \\
0 & 0 & 1 & 0 & 1
\end{array}\right), \\
A_{3}=\left(\begin{array}{lllll}
0 & 0 & 0 & 0 & 0 \\
0 & 0 & 0 & 0 & 0 \\
1 & 0 & 1 & 1 & 1 \\
0 & 0 & 0 & 0 & 0 \\
0 & 0 & 0 & 0 & 0
\end{array}\right),
\end{gathered}
$$

$I \in \operatorname{Mat}_{5}(\mathbb{Z})$ is the identity matrix, and $\overline{0} \in \operatorname{Mat}_{5}(\mathbb{Z})$ is the zero matrix.

Note that $A_{1}^{2}=\overline{0}$. We define $A_{4}=A_{3} A_{1}+A_{2}$ and $A_{5}=A_{1} A_{3}+A_{4}$, and find that in case $g=3$,

$$
S^{3}=\left(\begin{array}{ccc}
A_{5} & A_{1} & A_{3} \\
A_{2} A_{3} & A_{2} & A_{2} A_{1} \\
A_{3}+A_{2} A_{1} & A_{3} & A_{4}
\end{array}\right) .
$$

Furthermore, for $g \geq 4$, we claim that

$$
S^{g}=\left(\begin{array}{ccccccccccc}
A_{5} & A_{1} & \overline{0} & \overline{0} & \overline{0} & \overline{0} & \ldots & \overline{0} & \overline{0} & \overline{0} & A_{3} \\
A_{2} A_{3} & A_{2} & A_{2} A_{1} & \overline{0} & \overline{0} & \overline{0} & \ldots & \overline{0} & \overline{0} & \overline{0} & \overline{0} \\
A_{3} & A_{3} & A_{4} & A_{2} A_{1} & \overline{0} & \overline{0} & \ldots & \overline{0} & \overline{0} & \overline{0} & \overline{0} \\
\hline \overline{0} & \overline{0} & A_{3} & A_{4} & A_{2} A_{1} & \overline{0} & \ldots & \overline{0} & \overline{0} & \overline{0} & \overline{0} \\
\overline{0} & \overline{0} & \overline{0} & \overline{0} & \overline{0} & \overline{0} & \ldots & \overline{0} & A_{3} & A_{4} & A_{2} A_{1} \\
A_{2} A_{1} & \overline{0} & \overline{0} & \overline{0} & \overline{0} & \overline{0} & \ldots & \overline{0} & \overline{0} & A_{3} & A_{4}
\end{array}\right) .
$$

To show that, we choose $g \geq 4$, and define $c(i, n)$ as the $i$ th column of $S^{n}$, for $1 \leq i \leq g$ and $1 \leq n \leq g$. We will only demonstrate that $c(i, g)$ is as asserted for $i \in\{3, \ldots, g-1\}$. The remaining cases are similar.

Note first that $c(i, 1)$ has $I$ in its $(i-1)$ th position and zero matrices $\overline{0}$ in the other positions. We further compute

$$
\begin{aligned}
c(i, g-i) & =(\overline{0}, \ldots, \overline{0}, I), \\
c(i, g-i+1) & =(I, \overline{0}, \ldots, \overline{0}), \\
c(i, g-i+2) & =\left(A_{1}, A_{2}, A_{3}, \overline{0}, \ldots, \overline{0}\right), \\
c(i, g-i+3) & =\left(\overline{0}, A_{2} A_{1}, A_{4}, A_{3}, \overline{0}, \ldots, \overline{0},\right.
\end{aligned}
$$

and

$$
c(i, g)=\left(\overline{0}, \ldots, \overline{0}, A_{2} A_{1}, A_{4}, A_{3}, \overline{0}, \ldots, \overline{0}\right),
$$

where in the last equation $A_{2} A_{1}$ is in the $(i-1)$ th position as desired.

We now choose $g \in\{3,4, \ldots\}$, and note that as $\psi=\psi_{g}$ is p.A. with dilatation $\lambda=\lambda_{g}$, then $\psi^{g}$ is p.A. with dilatation $\lambda^{g}$. We showed in Step 1 that $\lambda$ is the spectral radius of the P.F. matrix $S$, hence $\lambda^{g}$ is the spectral radius of the P.F. matrix $S^{g}$. The spectral radius $\lambda^{g}$ of the P.F. matrix $S^{g}$ satisfies (see $[\mathrm{Ga}])$

$$
\lambda^{g}=\min \left\{\max _{1 \leq i \leq 5 g} \frac{\left(S^{g} x\right)_{i}}{x_{i}}: x \in \mathbb{R}^{5 g}, x>0\right\}
$$


and hence

$$
\lambda^{g} \leq \max _{1 \leq i \leq 5 g} \frac{\left(S^{g} x\right)_{i}}{x_{i}}
$$

for any $x \in \mathbb{R}^{5 g}, x>0$.

If we let

$$
\begin{gathered}
y_{1}=(.04, .22,1.2, .82, .28), \quad y_{2}=(.11, .65,1.47, .48, .8), \\
y_{3}=(.08, .47,2.2, .48, .52), \quad \text { and } x=\left(y_{1}, y_{2}, y_{3}, \ldots, y_{3}\right) \in \mathbb{R}^{5 g},
\end{gathered}
$$

then we compute that

$$
\frac{\left(S^{g} x\right)_{i}}{x_{i}} \leq 6, \quad \text { for } i=1, \ldots, 5 g .
$$

This completes the proof of the proposition. Q.E.D.

Remark. The estimate can be improved by better choice of $x$ in the proof of Proposition 5. Some values for $\left(\lambda_{g}\right)^{g}$ (as provided by Matlab) and

\begin{tabular}{c|c}
$g$ & $\left(\lambda_{g}\right)^{g}$ \\
\hline 3 & $\approx 5.50$ \\
4 & $\approx 5.35$ \\
5 & $\approx 5.28$ \\
6 & $\approx 5.25$ \\
9 & $\approx 5.21$
\end{tabular}

\section{REFERENCES}

[Ab] W. Abikoff, The real-analytic theory of Teichmüller space, Lecture Notes in Math., vol. 820, Springer-Verlag, 1980.

[AY] P. Arnoux and J. Yoccoz, Construction de difféomorphismes pseudo-Anosov, C. R. Acad. Sci. Paris 292 (1981), 75-78.

[Ba] M. Bauer, Examples of pseudo-Anosov homeomorphisms, Trans. Amer. Math. Soc. 330 (1992), 333-359.

[CB] A. Casson and S. Bleier, Automorphisms of surfaces after Nielson and Thurston, London Mathematical Soc. Student Texts 9, Cambridge Univ. Press, 1988.

[FLP] A. Fathi, F. Laudenbach, V. Poenaru et al., Travaux de Thurston sur les surfaces, Asterisque 66-67, Sem. Orsay, Soc. Math. de France, 1979.

[Ga] F. Gantmacher, Theory of matrices (vol. 2), Chelsea, 1960.

[HP] R. C. Penner (with J. L. Harer), Combinatorics of train tracks, Ann. of Math. Studies, Princeton Univ. Press, 1991.

[Pa] A. Papadopoulos, Difféomorphismes pseudo-Anosov et automorphismes symplectiques de l'homologie, Ann. Sci. Ecole Norm. Sup. 15 (1982), 543-546.

[P1] R. C. Penner, A construction of pseudo-Anosov homeomorphisms, Trans. Amer. Math. Soc. 130 (1988). 
[P2] - Bounds on least dilatations (to appear).

[PP] A. Papadopoulos and R. C. Penner, A characterization of pseudo-Anosov foliations, Pacific J. Math. 130 (1987).

[Th] W. Thurston, The geometry and topology of three-manifolds, Lecture Notes, Princeton Univ., 1978.

Department of Mathematics, University of ConNecticut, Storrs, ConNecticut 062693009

Current address: Département de Mathématiques, Université de Rennes I, Campus de Beaulieu, 35042 Rennes Cedex, France 